\title{
Gels in Ocean Waves - behaviour and implications for spill response
}

\author{
Richard J. Cunningham ${ }^{1}$, Hassan Sharifi ${ }^{2}$, Gregory A. Lawrence ${ }^{1}$ \\ ${ }^{1}$ Civil Engineering, University of British Columbia, Vancouver, Canada \\ ${ }^{2} \mathrm{BC}$ Research Inc, Vancouver, Canada
}

\begin{abstract}
While oil-gelling technology shows promise in the remediation of marine oil spills, the behaviour of marine surface gels has been left mostly unexplored in academia. In particular, the interactions between viscoelastic gels and surface waves are poorly understood. Determining what wave conditions result in the breakup of a gel layer could help predict the effectiveness of these technologies in the field.

This study adopts an experimental perspective to provide insight into the behaviour of viscoelastic gels in waves. Orbital motions of water particles in a linear wave will result in a spatially variable velocity field under a marine surface gel. This will cause different parts of the gel to experience drag of differing magnitude and direction, leading to a net tensile force which oscillates in time. Analytical models were developed to explain the response of simple elastic, Kelvin-Voigt, and Maxwell materials to this type of forcing.

While a low-density gel exposed to waves on the water surface is more directly comparable to oceanic conditions, dense-gel experiments could allow the isolation of wave-induced longitudinal stretching from wave-induced bending. In these experiments, a dense gel (gelatin) was placed on the bottom of a $4.7 \mathrm{~m}$ wave tank and constrained vertically. Applying monochromatic, linear waves to the system resulted in an observable oscillatory strain. Tests were conducted for a variety of gel lengths and wave conditions.

Breakage was observed when strain, and therefore stress, exceeded a certain threshold. The levels of stress required to achieve these strains were, however, greater than those predicted by the derived analytical models. This is likely the result of pressure effects associated with experimentation at the bottom of a wave tank. Nonetheless, this study provides an initial perspective on how wave-induced longitudinal stretching could influence oil-spill remediation.
\end{abstract}

Word count: 288 